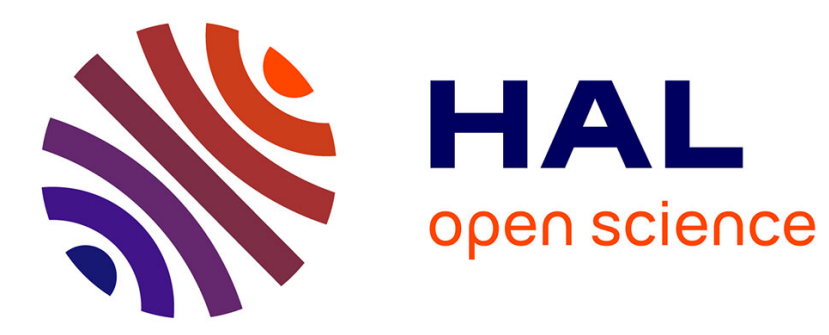

\title{
La faune de Mammifères du Pliocène terminal d'Ahl al Oughlam, Casablanca, Maroc
}

Denis Geraads, Fethi Amani, Jean-Paul Raynal, Fatima Zohra Sbihi-Alaoui

\section{To cite this version:}

Denis Geraads, Fethi Amani, Jean-Paul Raynal, Fatima Zohra Sbihi-Alaoui. La faune de Mammifères du Pliocène terminal d'Ahl al Oughlam, Casablanca, Maroc. Comptes rendus hebdomadaires des séances de l'Académie des sciences, 1998, 326, pp.671-676. halshs-00009695

\section{HAL Id: halshs-00009695 \\ https://shs.hal.science/halshs-00009695}

Submitted on 28 Mar 2006

HAL is a multi-disciplinary open access archive for the deposit and dissemination of scientific research documents, whether they are published or not. The documents may come from teaching and research institutions in France or abroad, or from public or private research centers.
L'archive ouverte pluridisciplinaire HAL, est destinée au dépôt et à la diffusion de documents scientifiques de niveau recherche, publiés ou non, émanant des établissements d'enseignement et de recherche français ou étrangers, des laboratoires publics ou privés. 
La faune de Mammifères du Pliocène terminal d'Ahl al Oughlam, Casablanca, Maroc

The Late Pliocene mammalian fauna of Ahl al Oughlam, Casablanca, Morocco

Denis GERAADS ${ }^{1}$, Fethi AMANI ${ }^{2}$, Jean-Paul RAYNAL ${ }^{3}$ et Fatima-Zohra SBIHI-ALAOUI ${ }^{2}$

1 UPR 2147 CNRS, 44 rue de l'Amiral Mouchez 75014 PARIS

2 Institut national des sciences de l'archéologie et du patrimoine, av.J.-F-Kennedy, Rabat-Souissi, Maroc

3 UMR 9933, Institut du Quaternaire, av. des Facultés, 33405 Talence cedex, France

\section{ABSTRACT}

The Late Pliocene site (ca $2.5 \mathrm{Ma}$ ) of Ahl al Oughlam has yielded a complete fauna of macro- and micro-mammals, by far the richest of the late Cenozoic era of North Africa,. it includes at least 55 species. Carnivora (23 species) are the dominant group. Many of them used the caves and fissures as dens or shelters, bringing in most of the ungulate remains. These remains include mostly medium-size species and juveniles of larger ones. Very few taxa are akin to Palearctic ones, and most of the faunal exchanges were intra-African. However; when compared to East Africa, the poor diversity of these ungulates must be noted, which the karstic nature of the site fails to fully explain, but which could result from a harsh, open and relatively cold environment.

Keywords: Mammals, Pliocene, Morocco, Faunal list

\section{RÉSUMÉ}

Le gisement du Pliocène final (environ 2,5 Ma) d'Ahl al Oughlam a livré une faune complète de microet macro-mammifères, de loin la plus riche de la fin du Cénozoïque d'Afrique du Nord, puisqu'elle comprend au moins 55 espèces. Elle est dominée par les Carnivores (23 espèces), dont beaucoup ont utilisé les grottes et fissures comme repaires, y apportant sans doute la plupart des restes d'Ongulés, parmi lesquels les formes de taille moyenne et les jeunes des espèces de grande taille sont majoritaires. Très peu de taxons ont des affinités avec des formes paléarctiques, et l'essentiel des échanges devait se faire avec le reste de l'Afrique. Cependant, comparativement à l'Afrique orientale, ces Ongulés sont remarquables par leur faible diversité, ce que la nature karstique du gisement ne suffit pas à expliquer, mais qui pourrait résulter d'un milieu peu favorable, ouvert et relativement froid.

Mots clés: Mammifères, Pliocène, Maroc, Liste faunique

\section{ABRIDGED VERSION}

The Ahl al Oughlam quarry is located at the southeastern limit of the city of Casablanca, $10 \mathrm{~km}$ from the present-day seashore. It was first mentioned in 1956 (Biberson, 1956, 1961). This author described, from a Messaoudian marine layer, broken pebbles that he took for man-made artefacts, but which are in fact of natural origin (Raynal et al. , 1990, 1995). A vertebrate assemblage was discovered in 1985 by Raynal and Texier from karstic and fissure fillings within continental deposits overlaying this marine fossil beach. The site has been worked from 1989 onwards by Geraads and colleagues as part of the 'Programme Casablanca' of the Institut National des Sciences de l'Archeologie et du Patrimoine, led by Sbihi-Alaoui and Raynal. Dated at more than $2 \mathrm{Ma}$, the fossil fauna demonstrates the Pliocene age of the classic Messaoudian transgression.

The vertebrate fauna is by far the richest of the North African Late Neogene, with about 80 species. Other than a few species of fish, amphibians, snakes and tortoises, it consists mainly of birds (under study by Mourer-Chauvire) and of more than 55 species of mammals, listed here (see also Geraads, 1997b). 
Primates. Theropithecus atlanticus (a species also known from Aïn Jourdel: Thomas, 1884, and somewhat different from the East and South African T.darti); Macaca sp. (the first known co-occurrence of this genus with the former). There is absolutely no evidence of the presence of hominids.

Carnivores. This is the most diverse group, with 23 species (Geraads, 1997a). They used the fissures as dens or shelters and brought in most of the ungulate remains. They include: Herpestes abdelalii (the most common carnivore, closely related to the living $H$. pulverulentus); Ichneumia nims (a rare species, more primitive than the recent sub-Saharan I. albicauda); Viverra leakeyi (also known at Omo); Genetta sp. ; Crocuta dbaa (a primitive spotted hyena, quite common); Pliocrocuta perrieri latidens (a species also known in Europe); Hyaenictitherium ? barbarum (survivor of a mainly Miocene group); Chasmaporthetes nitidula darelbeidae (hunting hyena, closely related to the South African form); Acinonyx aicha ( cheetah, not much different from the European A. pardinensis); cf. Dinofelis sp (fragmentary remains look closer to this genus than to Panthera); Panthera pardus (a small sized variety, as was usual at that time); Lynx sp. (a single bone); Felis cf. silvestris; Homotherium sp. (sabretoothed lion, rare); Nyctereutes abdeslami (the racoon dog, rare in Africa, is the most common Canidae here); Canis aff. aureus (a skull has recently been found); Vulpes aff. rueppelli (poorly known, but quite distinct from Eurasian forms); Prepoecilogale cf. bolti; Poecilictis libyca minor (both zorilla species are quite distinct from European mustelids); Mellivora cf. capensis; Lutra fatimazohrae; Ursus cf. etruscus (a few teeth demonstrate that the arrival of bears in North Africa occurred much earlier than previously thought); Alachtherium africanum (this is the southernmost known occurrence of a walrus, and the first in Africa).

Rodents (Geraads, 1995). Ahl al Oughlam is the only North African Late Neogene site rich in both micro- and macro-mammal faunas. Murids are similar to those from Irhoud Ocre and Bulla Regia (Jaeger, 1975) with Paraethomys chikeri; Praomys skouri oughlamensis and Mus haouzi. They are still more abundant than gerbils, represented by an early member of the genus Gerbillus, G. bibersoni. Irhoudia aff. bohlini and Hystrix sp. are quite rare.

Lagomorphs. Lepus sp. , cf. Serengetilagus sp. (a member of the Serengetilagus-Trischizolagus group, which survived until the Middle Pleistocene in this area; Geraads, 1994); Prolagus n.sp.

Insectivores. Consisting mostly of soricids, but Crocidura is still absent: Suncus barbarus, Episoriculus maghrehiensis; Erinaceus (Atelerix) sp .

Bats. At least two species can be found, but have not been studied as yet.

Proboscideans. This group is rare, and mostly from juvenile specimens: cf. Elephas sp. (perhaps $E$. recki); Anancus sp.

Cetaceans. Small stranded cetaceans were probably brought into the caves by carnivores: Delphinus or Stenella sp. (the most common taxon); Kogia sp. (a single petrosal, typical of the genus; Kasuya, 1973). Both genera live in warm or temperate waters.

Rhinocerotids. Ceratotherium mauritanicum (closely related to the living white rhinoceros, but longer limbed, perhaps better fitted to open environments, as already noted by Arambourg, 1970).

Equids. The absence of Equus, which appears in well-dated African sites by about $2.2 \mathrm{Ma}$, is the strongest argument for assigning an earlier age to Ahl al Oughlam. Hipparion cf. libycum is documented by one of the best Plio- Pleistocene samples of this genus.

Suids (Geraads, 1993). In contrast to East African sites, only one species, Kolpochoerus phacochoeroides, which is quite common, is present here and is somewhat distinct from K.limnetes. Camelids. cf. Camelus sp. , quite rare. The family is present in North Africa from the Early Pliocene onwards (Arambourg,1979).

Giraffids (Geraads, 1996). Once again in contrast to East Africa, there is no Giraffa, but only Sivatherium maurusium.

Bovids. They are similar to East African bovids, although most of them are distinct at the specific level (Geraads and Amani, in press): Tragelaphus sp. (tragelaphines are much less common than in East Africa); Bovini indet. (perhaps close to Pelorovis); Kobus n.sp. (a short-horned species, perhaps of an 
endemic lineage); Beatragus antiquus n.subsp. (the genus was previously known from East Africa only); Parmularius sp. (a widespread African genus); Gazella n.sp. (a strange species, with nasal adaptations reminiscent of the Saiga antelope);

\section{Gazella thomasi.}

Although Ahl al Oughlam is roughly contemporaneous with several famous hominid-bearing East African sites, it has not yielded the slightest evidence of the presence of hominids. We believe that this absence is significant, because of the richness of the site. We may therefore take for granted that our ancestors had not reached that part of the continent by the Late Pliocene. However, the fauna as a whole is rather similar to that in East and South Africa. Ahl al Oughlam shares several species, and most of its genera, with these areas. Only a few taxa are clearly of palearctic origin, and unknown in the rest of Africa. Endemic taxa are still less numerous. It is clear, therefore, that northern influences were of limited impact, and that most of the exchanges were intra-African, implying that there was certainly no continuous Saharian barrier. However, the poor faunal diversity, especially among large ungulates, is in sharp contrast to East Africa. We may exclude any significant taphonomic bias, but this poor diversity may be partly explained by the accumulation process, which sampled a single biocenosis, in contrast to open-air East African sites, where fossils from various environments may be mixed. We believe, however, that this factor alone fails to fully account for the poor diversity at Ahl al Oughlam, the environment of which must have been rather harsh. There are indicators that it was open, but reduncines show that it was not very dry; in fact, it was certainly cooler than in East Africa, and this temperate climate might have allowed the settlement of northern taxa such as Macaca, Ursus, Alachtherium and Gazella n.sp.

\section{Introduction}

La carrière d'Ahl al Oughlam est située à environ $10 \mathrm{~km}$ du rivage actuel, au bord de la route menant de Casablanca à Tit Mellil. Le site a été signalé dès 1956 par Biberson sous le nom de «carrière Déprez ». Cet auteur crut y reconnaître $(1956,1961)$ les plus anciens témoignages au Maroc de la « Pebble Culture », sous forme de galets fracturés provenant d'un niveau marin attribué au Messaoudien. Il ne s'agit en fait que de cassures naturelles dues à l'action hydraulique (Raynal et al., 1990 ; 1995). Le site a été réexaminé à partir de 1985 par J .-P. Raynal et J.-P. Texier dans le cadre des recherches francomarocaines menées dans cette région. A cette occasion, une petite faune terrestre fut recueillie dans des niveaux continentaux postérieurs au maximum transgressif messaoudien. En 1989 et 1990, de nouvelles récoltes sont venues enrichir les collections (actuellement déposées au musée archéologique de Rabat). Elles nous ont permis (Raynal et al., 1990) de dresser une première liste faunique, et de proposer pour cet assemblage un âge supérieur à $2 \mathrm{Ma}$, établissant ainsi clairement l'âge pliocène du « Messaoudien » classique.

Depuis lors, la poursuite de la fouille du gisement, menée par D.G. dans le cadre du « Programme Casablanca » de l'Institut national des sciences de l'archéologie et du patrimoine (Insap), dirigé par F.Z. S.-A. et J.-P. R. (CNRS), a conduit à la mise au jour de plusieurs milliers de fossiles, appartenant à près de 80 espèces de Vertébrés, faisant du gisement d'Ahl al Oughlam le plus riche de tout le Néogène nordafricain.

La totalité du matériel provient d'un réseau de fissures et de galeries interconnectées dans un effondrement de blocs de calcarénite, au pied d'un rivage fossile. Le remplissage est d'origine clastique et karstique. Le sédiment peut être sableux, ou au contraire extrêmement bréchifié, et la taille et l'état de conservation des fossiles varient suivant les zones fossilifères, mais aucune stratigraphie de détail n' est apparente, et le remplissage peut assurément être considéré comme instantané à l'échelle géologique. En dehors de quelques espèces de Poissons (Requins et Sparidés), d'Amphibiens, de Squamates (en cours d'étude par Bailon) et de Tortues, ce sont surtout les Oiseaux (une vingtaine d'espèces, en cours d'étude par Mourer-Chauviré) et les Mammifères qui constituent l'essentiel de la faune. L'étude de ce 
dernier groupe est aujourd'hui suffisamment avancée pour qu'une liste faunique à peu près complète puisse en être établie. Des détails sur de nombreux taxons sont accessibles par ailleurs (Geraads, 1997b).

2. Systématique

\subsection{Primates}

Ils ne comprennent que deux genres de Cercopithecidés (Alemseged et Geraads, sous presse), dont la coexistence est ici démontrée pour la première fois :

-Macaca sp. Trois dents seulement sont rattachées à ce genre ;

-Theropithecus atlanticus (Thomas, 1884) ; cette espèce endémique, également connue à l' Aïn Jourdel (Thomas, 1884), est un peu différente de T.darti d'Afrique orientale et méridionale.

Aucune trace de présence ou d'activité humaine n'a pu être mise en évidence.

\subsection{Carnivores}

Les Carnivores constituent de loin le groupe de Mammifères le plus diversifié, et ils sont aussi très abondants, en nombre de spécimens, par rapport aux Herbivores, sans doute parce qu'ils utilisaient les fissures comme abris ou tanières (Geraads, 1997a) :

-Herpestes abdelalii Geraads, 1997 ; un peu plus primitive que l'actuelle $H$. pulverulentus, cette mangouste est le carnivore le plus abondant;

-Ichneumia nims Geraads, 1997 ; cette autre mangouste, représentée par deux carnassières inférieures, est plus primitive que l'actuelle I. albicauda, aujourd'hui inconnue au nord du Sahara ;

-Viverra leakeyi Petter, 1963, connue par une unique dent, semblable à celles d'Afrique de l'Est, est proche de la civette africaine ;

-Genetta sp. ; la genette n' est, elle aussi, représentée que par une seule dent ;

-Crocuta dbaa Geraads, 1997, est une hyène intermédiaire entre C. dietrichi de Laetoli et C. crocuta ; c'est le plus fréquent des grands Carnivores ;

-Pliocrocuta perrieri latidens Geraads, 1997 semble voisine de la forme européenne, mais les relations phylétiques dans ce groupe sont encore incertaines ;

-Hyaenictitherium barbarum Geraads, 1997, dont de nouveaux spécimens ont été récemment découverts, semble être un survivant d'un groupe surtout connu au Miocene ;

-Chasmaporthetes nitidula darelbeidae Geraads, 1997, est une hyène félinoïde voisine de la forme sudafricaine ;

-Acinonyx aicha Geraads, 1997, est un guépard un peu différent de A. pardinensis d'Europe.

-cf. Dinofelis sp. ; c'est de ce genre qu'il convient en fait de rapprocher le grand félidé qui avait été comparé à Panthera leo (cf. Geraads, 1997a), mais qui n'est encore représenté que par des restes très fragmentaires ;

-Panthera pardus (L.) est un léopard de petite taille, comme la plupart des formes anciennes de cette espèce ;

-Lynx sp. n'est représenté que par un seul os ;

-Felis sp., cf. silvestris Schreber, 1777 ;

-Homotherium sp. ; ce félidé à canines en sabre, de la taille d'un lion, n'est représenté ici que par quelques dents; le genre survit en Afrique du Nord jusqu'au milieu du Pléistocène ;

-Nyctereutes abdeslami Geraads, 1997 ; ce genre, fréquent en Eurasie, n' avait jusqu'alors été signalé que dans le Sud du continent africain; c'est ici le canidé le plus abondant;

-Canis nov. sp., aff. C. aureus L. ; un crâne, récemment découvert, permettra de préciser les affinités de cette espèce voisine du chacal ;

-Vulpes sp., aff. rueppelli Schinz, 1825, est sans doute aussi une espèce nouvelle, mais demeure mal connue ;

-Prepoecilogale sp., cf. bolti (Cooke, 1985);

-Poecilictis libyca minor Geraads, 1997 ; ces deux espèces de zorilles sont bien différentes des

Mustélidés européens ;

-Mellivora sp., cf. M. capensis (Schreber, 1776) ; 
-Lutra fatimazohrae Geraads, 1997 ;

-Ursus sp., cf. U. etruscus Cuvier, 1823 ; les quelques dents d'Ahl al Oughlam démontrent que l'arrivée des ours en Afrique du Nord est bien antérieure à ce qu'on pensait naguère; par la suite, d'autres échanges semblent avoir eu lieu, entre des phases d'isolement ayant conduit à au moins une espèce endémique ;

-Alachtherium africanum Geraads, 1997. Les morses habitent aujourd'hui les mers arctiques, où la chasse les a repoussés, mais aux temps historiques et préhistoriques ils s'étendaient au sud jusqu'en Belgique; Ahl al Oughlam, où ils sont représentés par plusieurs individus, jeunes et adultes, reste cependant de loin leur mention la plus méridionale, et la première en Afrique.

2.3. Rongeurs

Des milliers de dents et de fragments de mâchoires de Rongeurs ont été récoltés par tamisage (Geraads, 1995). Ahl al Oughlam est le seul site de la fin du Néogène d'Afrique du Nord ayant livré une faune riche à la fois en micro- et macro-mammifères :

-Paraethomys chikeri Jaeger, 1975 ;

-Praomys skouri oughlamensis Geraads, 1995 ;

-Mus haouzi Jaeger, 1975 (ces trois Muridés sont voisins de ceux décrits par Jaeger (1975) à Irhoud Ocre et Bulla Regia ; ils dominent encore largement les Gerbillidés, contrairement à ce qu'on observe plus tard dans la même région, sans doute à la suite d'une dégradation climatique) ;

-Gerbillus bibersoni Geraads, 1995, est déjà très proche de l'actuelle G. campestris, bien qu'il s'agisse de l'un des plus anciens représentants de ce genre ;

-Irhoudia sp., aff. I. bohlini jaeger, 1971;

-Hystrix sp.

2.4. Lagomorphes

Les lièvres et lapins sont en cours d'étude. Particulièrement intéressant est un représentant du groupe Serengetilagus-Trischizolagus, également présent dans le Pléistocène moyen de la même région

(Geraads, 1994) :

-Lepus sp. ;

-cf. Serengetilagus sp.;

-Prolagus n. sp.

2.5. Insectivores

Ils consistent presque exclusivement en Soricidés (Geraads, 1995), mais il faut noter l'absence de Crocidura, qui deviendra l'unique genre de la région au Pléistocène :

-Suncus barbarus Geraads, 1995 ;

-Episoriculus maghrebiensis Rzebik-Kowalska, 1988 ;

-Erinaceus (Atelerix) sp.

2.6. Chiroptères

Il y a au moins deux espèces de chauve-souris, non encore étudiées.

2.7. Proboscidiens

Deux espèces sont représentées par quelques os des membres et des dents de jeunes individus :

-cf. Elephas sp., semble voisin de E. recki, bien connu en Afrique orientale, et est sans doute ancestral à l'Elephas iolensis d'Afrique du Nord;

-Anancus sp., mastodonte à défenses droites, connu également en Europe et Afrique orientale par des formes peut-être un peu différentes (Metz-Mueller, in litt.).

2.8. Cétacés

De petits Cétacés échoués ont probablement été apportés dans les grottes par les carnivores :

-Delphinidé gen. et sp. indét., Delphinus sp. ou Stenella sp. (une douzaine de spécimens, pétreux et fragments de mâchoires) ;

-Kogia sp. : le cachalot pygmée est représenté par un pétreux, bien caractéristique (Kasuya, 1973).

Ces deux Cétacés, contrairement aux morses, habitent plutôt les eaux chaudes ou tempérées. 


\subsection{Rhinocérotidés}

-Ceratotherium sp., cf. C. mauritanicum (Pomel, 1895), cousin du Rhinocéros blanc, connu également en Afrique orientale, et peut-être mieux adapté aux milieux ouverts par ses métapodes allongés, comme l'avait noté Arambourg (1970).

\subsection{0. Équidés}

Equus est absent à Ahl al Oughlam. Comme il apparaît dans l' Ancien Monde avant $2 \mathrm{Ma}$, et dans des sites est-africains bien datés autour de 2,2 Ma, Ahl al Oughlam doit être plus ancien.

-Hipparion sp., cf. H. libycum Pomel, 1897. Ahl al Oughlam a livré l'un des plus importants échantillons plio-pléistocènes de ce genre (plusieurs dizaines de métapodes, quelques séries dentaires, un crâne complet bien qu'écrasé) .

\subsection{Suidés}

Kolpochoerus phacochoeroides (Thomas, 1884). Les Suidés ne sont représentés que par une seule espèce, très commune, un peu différente des formes est-africaines (Geraads, 1993).

2.12. Camélidés

Cf. Camelus sp., très rare. Les Camélidés apparaissent en Afrique du Nord, au début du Pliocène, au lac Ichkeul (Arambourg, 1979), mais demeurent rares avant le milieu du Pléistocène.

\subsection{Giraffidés}

Sivatherium maurusium (Pomel, 1893). Dans cette famille également, la diversité est bien moindre qu'en Afrique orientale, où Sivatherium coexiste généralement avec Giraffa, absent ici (Geraads, 1996).

2.14. Bovidés

Les Bovidae présentent d'étroites affinités avec ceux d'Afrique orientale, bien que la plupart d'entre eux soient différents au niveau spécifique (Geraads et Amani, sous presse) :

-Tragelaphus sp. est un koudou représenté par quelques dents seulement, peut-être semblable à $T$. gaudryi de l' Aïn Jourdel ; la rareté des Tragelaphini contraste avec leur abondance en Afrique orientale ; -Bovini indet. ; les Bovini nord-africains ne sont illustrés que par des pièces incomplètes, mais on peut sans doute les rapprocher de Pelorovis;

-Kobus n. sp. est un cobe à cornes courtes, peut-être d'une lignée endémique ;

-Beatragus antiquus n. subsp ; ce genre de damalisque n' était jusque-là connu qu'en Afrique orientale ;

-Parmularius n. sp. ; Parmularius est un genre éteint largement répandu dans tout le Plio-Pléistocène d'Afrique;

-Gazella n. sp. ; cette gazelle est remarquable par ses adaptations nasales rappelant la saïga et le dik-dik ; -Gazella thomasi (Pomel, 1895).

3. Conclusions

Bien que le site soit à peu près contemporain des célèbres gisements est-africains de l'Omo, de la partie supérieure de Hadar et du Middle Awash (Ethiopie), ou de l'Est et de l'Ouest Turkana (Kenya), il n'a pas livré la moindre trace de présence humaine. Nous pensons que cette absence est significative, compte tenu de la richesse du site. Il est donc à peu près certain que nos ancêtres n'avaient pas encore conquis cette partie du continent à la fin du Pliocène. Pourtant, la faune dans son ensemble est assez semblable à celles d'Afrique orientale et méridionale; plusieurs espèces sont communes, et la presque totalité des genres le sont également. Parmi les taxons terrestres, seuls quelques-uns sont clairement d'origine paléarctique: Macaca, Episoriculus, Ursus et Nyctereutes. À l'exception du dernier, ils sont aussi inconnus en Afrique orientale et méridionale. Les genres endémiques à la région à cette époque sont rarissimes: Poecilictis (mais les petits carnivores sont mal connus dans le reste de l' Afrique),

Paraethomys, lrhoudia. Il est donc clair que les influences paléarctiques sont restées très marginales, et que la presque totalité des échanges fauniques s'effectue alors aisément avec le reste de l' Afrique, impliquant l'absence de barrière saharienne continue. Pourtant, la faible diversité de la faune, surtout sensible chez les grands Ongulés, contraste avec la richesse observée dans la plupart des sites estafricains de cet âge. La grande quantité d'ossements recueillie permet d'exclure toute explication taphonomique basée sur l'imperfection de l'échantillonnage opéré par les carnivores ou les fouilleurs. 
Même si cet échantillonnage n'est pas exhaustif, cette faible variété reflète donc sans aucun doute celle de la biocénose. Il se peut qu'elle soit en partie due à l'homogénéité de l'assemblage faunique, à la différence des sites de plein air, où les faunes issues de plusieurs biotopes peuvent être mêlées, accroissant artificiellement la biodiversité. Ce seul facteur est cependant insuffisant, et il faut donc aussi faire intervenir la faiblesse de la productivité végétale. L'abondance des Alcélaphinés, des gazelles, la rareté des Primates, sont de bons indicateurs d'un milieu ouvert, mais la fréquence des cobes montre aussi qu'il n'était certainement pas très sec. On peut penser que le climat était pourtant moins favorable qu'en Afrique orientale, et sans doute plus froid. Ce climat tempéré a sans doute favorisé l'installation de genres tels que Macaca, Ursus, Alachtherium, et Gazella n. sp.

\section{RÉFÉRENCES}

Alemseged Z. et Geraads D. Theropithecus atlanticus (Cercopithecidae, Mammalia) from the Late Pliocene of Ahl al Oughlam, Casablanca, Morocco, J. Hum. Evol. (sous presse)

Arambourg C. 1970. Les Vertébrés du Pléistocène de l' Afrique du Nord, Arch. Mus. Nat. Hist. Nat., sér.7, 10,1-126

Arambourg C. 1979. Vertébrés villafranchiens d'Afrique du Nord (Artiodactyles, Carnivores, Primates, Reptiles, Oiseaux), Fondation Singer-Polignac, Paris, 1-141.

Biberson P. 1956. Nouvelles précisions sur les gisements à Pebble-Culture des plages marines soulevées du Quaternaire ancien de Casablanca (Maroc), C. R. Acad. Sci. Paris, 243, 1227-1229

Biberson P. 1961. Le cadre paléogéographique de la Préhistoire du Maroc Atlantique, Publ. Serv. Antiq. Maroc, 16,1-235

Geraads D. 1993. Kolpochoerus phacochoeroides (Thomas, 1884) (Suidae, Mammalia), du Pliocène supérieur de Ahl al Oughlam (Casablanca, Maroc), Géobios, Lyon, 26 (6),731-743

Geraads D. 1994. Rongeurs et Lagomorphes du Pléistocène moyen de la «Grotte des Rhinocéros », carrière Oulad Hamida I à Casablanca, Maroc, N. Jb. Geol. Paläont. Abh., 191 (2) 147-172

Geraads D. 1995. Rongeurs et Insectivores du Pliocène final de Ahl al Oughlam, Casablanca, Maroc, Géobios, Lyon, 28 (1 )1 99-115

Geraads D. 1996. Le Sivatherium (Giraffidae, Mammalia) du Pliocène final d'Ahl al Oughlam (Casablanca, Maroc) et l'évolution du genre en Afrique, Paläont. Zeitschr., 70 (3-4),623-629

Geraads D. 1997a. Carnivores du Pliocène terminal de Ahl al Oughlam (Casablanca, Maroc), Géobios, Lyon, 30 (1),127-164

Geraads D. 1997b. Ahl al Oughlam, un gisement de Vertébrés du Pliocène final du Maroc,

Geraads D. et Amani F. Bovidés du Pliocène final d'Ahl al Oughlam (Casablanca, Maroc), Paläont. Zeitschr. (sous presse)

Jaeger j.-j. 1975. Evolution des Rongeurs, du Miocène à 1 'actuel, en Afrique nord-occidentale, Thèse, USTL, Montpellier

Kasuya T. 1973. Systematic consideration of recent toothed whales based on the morphology of tympano-periotic bone, Sci. Rep. whales Res. Inst., Tokyo, 25,1-103

Raynal J.-P. Texier J.-P. 1 Geraads D. et Sbihi-Alaoui F.Z. 1990.- Un nouveau gisement paléontologique du Plio-Pléistocène du Maroc: Ahl al Oughlam (ancienne carrière Deprez), C. R. Acad. Sci. Paris, 310 , série Il, 315-320

Raynal J.-P. Magoga L., Geraads D. et Sbihi-Alaoui F.Z. 1995. The earliest occupation of Atlantic Morocco: the Casablanca evidence, in: Roebroeks W. et van Kolfschoten T. (éds), The earliest occupation of Europe, University of Leiden, 13,255-262

Thomas P. 1884. Recherches stratigraphiques et paléontologiques sur quelques formations d'eau douce de l' Algérie, Mém. Soc. géol. France, série 3,3 (2),1-50 